\title{
MOBILITY AND SESSION MANAGEMENT: UMTS VS. CDMA2000
}

\author{
Al-Chun Pang, National taiwan University \\ JyH-Cheng Chen, National Tsing Hua University \\ Yuan-Kal Chen, Chunghwa Telecom Co., Ltd. \\ Prathima AgraWal, Telcordia TeChNologies
}

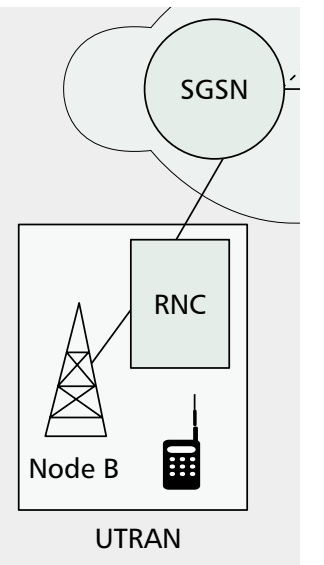

UMTS and cdma2000

are two major standards

for $3 G$ mobile

telecommunication or

IMT2000. Many

operators commit to

deploy UMTS and/or

cdma2000-based $3 G$

networks. Evolving from

the existing $2 G$

networks, construction

of effective $3 G$ network

is critical for provisioning

future mobile services.

\section{ABSTRACT}

This article describes the mobility and session management mechanisms for UMTS and cdma2000 packet-switched (PS) service domains, and compares the design guidelines for these two third-generation technologies. We first introduce the network architectures and protocols for UMTS and cdma2000, and then elaborate on the PS service domain's mobility management, session management, and IP-level mobility mechanisms. Based on the mobility and session management mechanisms of the UMTS and cdma2000 PS service domains, an integrated architecture and intersystem roaming procedures are proposed to show the implementation feasibility of UMTS-cdma2000 IP-level interworking.

\section{INTRODUCTION}

Universal Mobile Telecommunication System (UMTS) and cdma2000 (based on code-division multiple access, CDMA) are two major standards for third-generation (3G) mobile telecommunication or IMT2000 [1-4]. Many operators commit to deploying UMTS and/or cdma2000based $3 \mathrm{G}$ networks. Evolving from existing $2 \mathrm{G}$ networks, construction of an effective $3 \mathrm{G}$ network is critical for provisioning future mobile services. This article describes two important functionalities of $3 \mathrm{G}$ networks, mobility management and session management, and compares their design guidelines for UMTS and cdma2000. We focus on discussions and comparisons for the UMTS and cdma2000 packet-switched (PS) service domains. The reader is referred to [5] for interoperability between the circuit-switched (CS) service domains of UMTS and cdma2000.

The mobility management functions are used to keep track of the current location of a mobile user. There are three types of mobility: radio mobility, core network (CN) mobility, and IP mobility. Radio mobility supports handoff (i.e., radio link switching) of a mobile user during conversation. CN mobility (or link layer mobility) provides tunnel-related management for packet rerouting in the $\mathrm{CN}$ due to user movement. The IP mobility mechanism allows the mobile user to change its access point of IP connectivity without losing ongoing sessions. Network nodes such as the gateway GPRS support node (GGSN) and home agent (HA; their functions will be elaborated later) hide the mobility of the mobile user from the external network so that the corresponding user (communicating with the mobile user) is not notified.

Session management maintains the routing path for a communication session between a mobile user and the $3 \mathrm{G} \mathrm{CN}$, which provides packet routing functions, including IP address assignment, quality of service (QoS) setting, and so on. Details of mobility and session management mechanisms for UMTS and cdma2000 will be elaborated on later. In the remainder of this section we briefly describe the UMTS and cdma2000 network architectures, and explain the roles of different nodes regarding mobility and session management.

UMTS is evolved from the Global System for Mobile Communications (GSM) and General Packet Radio Service (GPRS). The network architecture of UMTS (Release 99) is shown in Fig. 1 [6]. In this figure, the dashed lines represent signaling links, and the solid lines represent data and signaling links. The CN consists of two service domains, a CS service domain and a PS service domain. In the CS service domain, UMTS connects to the public switched telephone network (PSTN) through a mobile switching center (MSC). In the PS service domain, UMTS connects to the packet data network (PDN) through the serving GPRS support node (SGSN) and GGSN. The SGSN in the PS service domain is "equivalent" to the MSC in the CS service domain. The GGSN provides interworking with external PDNs, and is connected with SGSNs via an IP-based GPRS backbone network. Both SGSN and GGSN provide session management functions in a data communication session. Mobility management is exercised between the SGSN and the home location register (HLR). The UMTS terrestrial radio access network (UTRAN) consists of Node Bs 


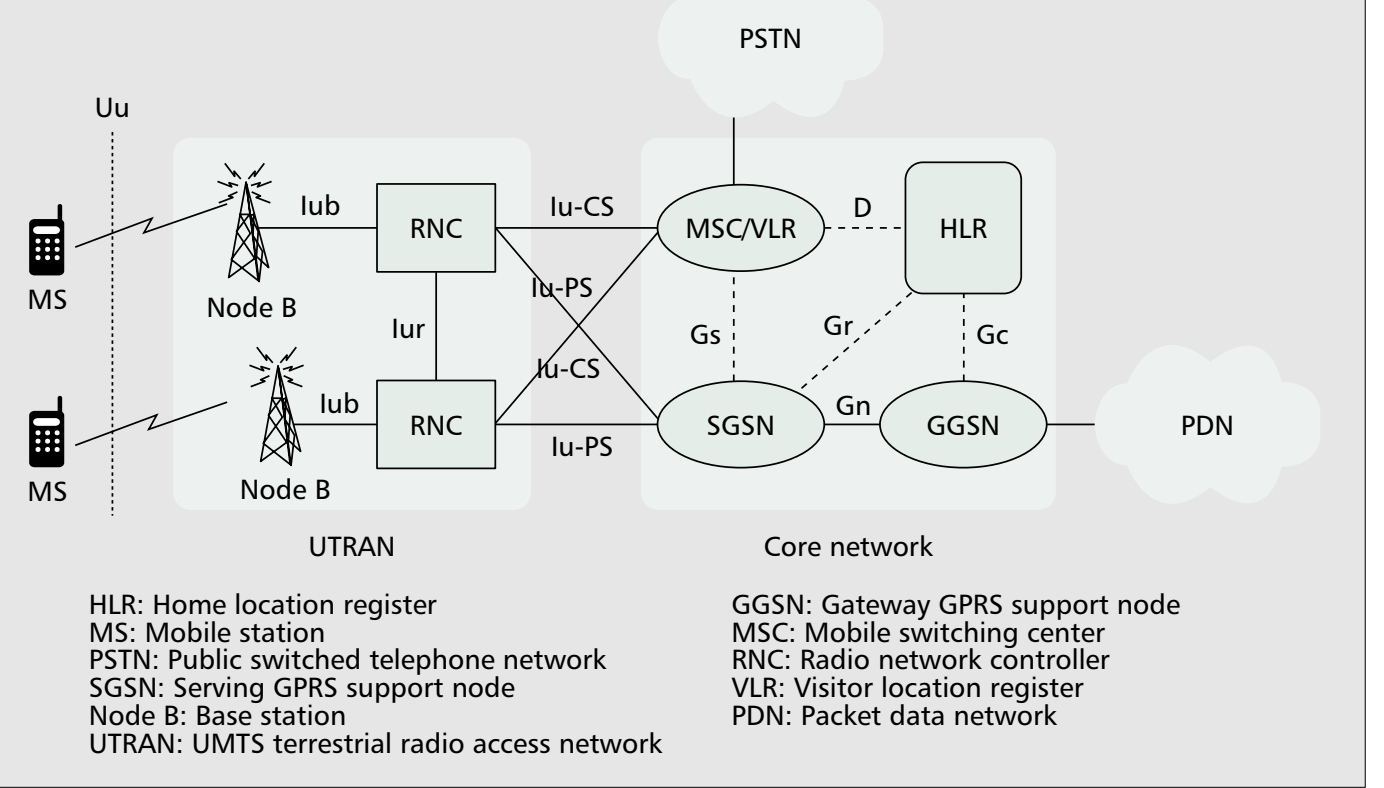

Figure 1. UMTS network architecture.
GGSN provides

interworking with external PDN, and is connected with SGSNs via an IP-based GPRS backbone network. Both

SGSN and GGSN provide session management functions in a data communication session.

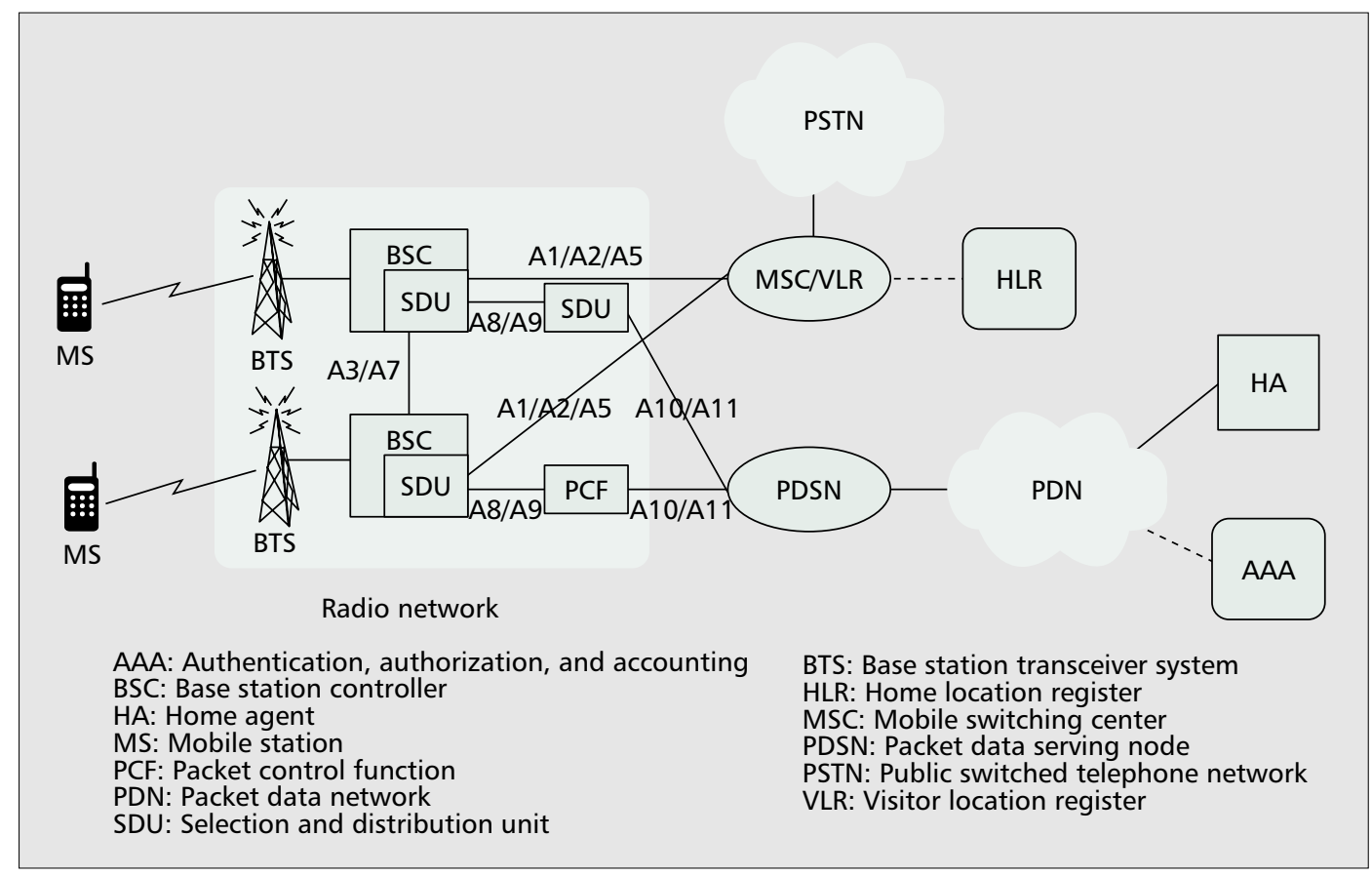

Figure 2. cdma2000 architecture.

(the UMTS term for base stations) and radio network controllers (RNCs) connected by an asynchronous transfer mode (ATM) network. The interface between a Node B and an RNC is Iub. The interface between two RNCs is Iur; it supports soft handoff. An RNC connects to an MSC and an SGSN through the Iu-CS and Iu-PS interfaces, respectively. These Iu interfaces are based on ATM technologies. The mobile station (MS) or user equipment connects with Node Bs through the radio interface $U u$ based on wideband CDMA (WCDMA) radio technology [7].

In UMTS, the Mobile Application Part (MAP) provides mobility management interfaces between the SGSN and the GSM network nodes; for example, Gr for the HLR and Gs for the MSC/visitor location register (VLR). SGSNs and GGSNs communicate by using the GPRS Tunneling Protocol (GTP) through the Gn interface [8]. Details of Gr, Gs, and Gn can be found in [6]. We will elaborate on GTP later.

Figure 2 shows the cdma2000 architecture [9]. In this figure, the base station controller (BSC) connects to the $\mathrm{CN}$ through the selection and distribution unit (SDU). Like UMTS, cdma2000 supports both the CS and PS service domains. The SDU distributes the CS traffic (e.g., voice) to the MSC via interfaces A1, A2, and A5. 
The control plane carries

out tasks for mobility

management, session

management, and short

message service. The

mobility and session

tasks in cdma2000 are

based on the same

lower layer protocols for

user data transportation

except that the user

data flow bypasses

the LAC layer.

${ }^{1}$ In cdma2000, user and device authentication functions are handled by the MSC/VLR/HLR. In UMTS, the SGSN directly interacts with the HLR to exercise user, device, and service authentication.

2 In UMTS, the UTRAN utilizes the direct transfer procedure for handling mobility and session management messages exchanged between the $M S$ and SGSN. The direct transfer procedure relays these messages between the MS and SGSN by converting the message formats without interpreting the contents of the messages. In cdma2000 these messages are delivered using a PPP session (between the MS and PDSN), where the RN is not involved in format translation of these messages.
Specifically, the A1 interface supports call control and mobility management between the MSC and BSC. The A2 and A5 interfaces support voice traffic and CS data traffic between the BSC and MSC, respectively. The MSC, VLR, and HLR functions are basically the same as those in UMTS, which will not be re-elaborated. The SDU distributes PS traffic to the packet control function (PCF) and then to the packet data serving node (PDSN). Interfaces A8 and A9 support PS data and signaling between the PCF and SDU, respectively. Interfaces A10 and A11 (i.e., the R-P interface) support PS data (which is delivered in sequence) and signaling between the PCF and PDSN. The generic routing encapsulation (GRE) tunnel [10] with standard IP QoS is used for data routing in A10, and A11 utilizes Mobile IP (MIP) [10, 11] based messages to convey the signaling information between the PCF and PDSN. The R-P interface (i.e., A10/A11 interface) also supports PCF handoff (inter- or intra-PDSN), described later.

A PDSN connects to one or more BSCs, which establishes, maintains, and terminates link layer sessions to the MS. The PDSN supports packet compression and packet filtering (see the Pointto-Point Protocol, PPP, description below) before the packets are delivered through the air interface. The PDSN provides IP functionality to the mobile network, which routes IP datagrams to the PDN with differentiated service support. A PDSN interacts with authentication, authorization, and accounting (AAA) [12] to provide IP authentication, authorization, and accounting support for packet data services (note that user and device authentication functions are not provided in the PDSN $^{1}$ ). The PDSN may act as an MIP foreign agent (FA) in the mobile network, which provides mobility management mechanisms with the MIP HA. The interfaces among the PDN nodes (i.e., PDSN, HA, AAA) follow Internet Engineering Task Force (IETF) standards.

From the above discussions, the network architectures of mobility and session management are basically the same for both UMTS and cdma2000. However, the protocols exercised on these network architectures are different. For example, cdma2000 uses IETF protocols (e.g., MIP and PPP) to support mobility and session management mechanisms. On the other hand, the UMTS protocols include SS7-based MAP and IP-based GTP. Moreover, one of the key 3G goals is to provide independence between the radio and core networks. As we shall see in the following sections, UMTS provides clear demarcation between the RAN and CN. On the other hand, this goal is only partially achieved in cdma2000, where the MSC is still involved in radio resource allocation for a packet session.

The remainder of this article is organized as follows. The following section describes the protocol stacks that support mobility and session management for cdma2000 and UMTS. Next, the mobility and session management mechanisms for cdma2000 and UMTS are presented and compared. Then IP mobility supported in cdma2000 and UMTS is elaborated on. The architecture and algorithms for UMTScdma2000 IP-level interworking are proposed. Finally, a summary is given.

\section{Protocol StaCKS}

In this section we briefly discuss the lower layer protocols that support mobility and session management and user data transport. Figures 3 and 4 illustrate the control planes and user planes for cdma2000 and UMTS networks, respectively.

The control plane carries out tasks for mobility management, session management, and short message service. The mobility and session tasks in cdma2000 are based on the same lower layer protocols (i.e., IP-based protocols; Figs. 3a and 4a) for user data transportation except that the user data flow bypasses the link access control (LAC) layer. An advantage of the cdma2000 approach is that the same lower layer protocols can support both control and user planes.

In UMTS, the lower layer protocols supporting these tasks in the control plane (Fig. 3b) are different from those in the user plane (Fig. 4b). The UMTS control plane protocols such as radio resource control (RRC) and RAN Application Part (RANAP) are utilized for signaling. Specifically, the signaling path between MS and SGSN consists of an RRC connection between MS and UTRAN, and an Iu connection ("one RANAP instance") between UTRAN and SGSN. The $\mathrm{RRC}$ protocol is responsible for reliable connection between MS and UTRAN; that is, radio resources are managed by $\mathrm{RRC}$ exercised between MS and UTRAN. The Signaling Connection Control Part (SCCP) is responsible for reliable connection between UTRAN and SGSN. On top of SCCP, the RANAP protocol supports transparent non-access stratum (e.g., mobility management, session management) signaling transfer between MS and CN, which are not interpreted by the UTRAN. ${ }^{2}$ By using these signaling protocols, efficient radio resource management, session management, and mobility management can be achieved. In cdma2000 the task of radio resource management involves the MSC [10]. That is, the MSC instructs the BSC to assign the radio resource through the A1 interface. The details are for further study in cdma2000 specifications.

In UMTS, the PS domain services are supported by the Packet Data Convergence Protocol (PDCP) in the user plane. The PDCP contains compression methods needed to provide better spectral efficiency for IP packet transmission over radio. In cdma2000, the header and payload compression mechanism is provided by PPP between the MS and PDSN.

Both the UMTS radio link control (RLC) protocol (Figs. 3b and 4b) and cdma2000 LAC protocol (Figs. 3a and 4a) provide segmentation and retransmission services for user and control data. In addition, the cdma2000 LAC protocol supports the authentication functionality for wireless access, which is equivalent to GPRS transport layer authentication in UMTS. In the remainder of this section we elaborate on the protocols regarding IP support and tunneling.

\section{PPP}

In both the control and user planes for cdma2000, PPP is carried over the LAC/MAC and R-P tunnels (to be elaborated on later) and utilized to establish connection between the MS 


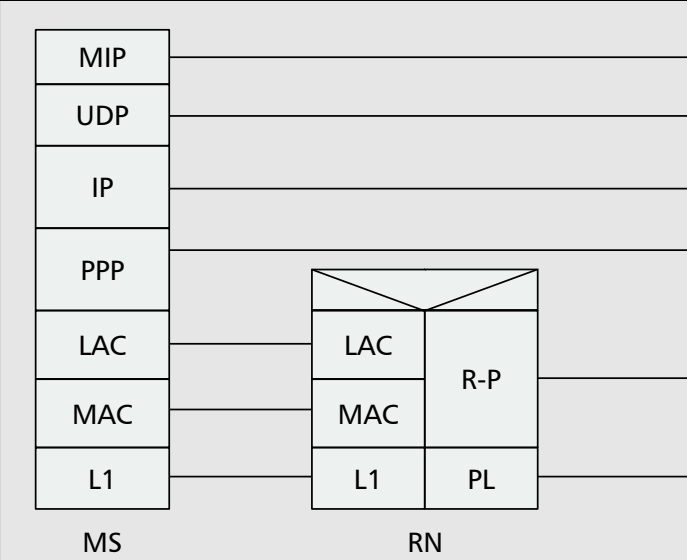

IKE: Internet key exchange IPSec: IP security LAC: Link access contro MIP: Mobile IP

PDSN: Packet data serving node PL: Physical layer

R-P: RN-PDSN interface

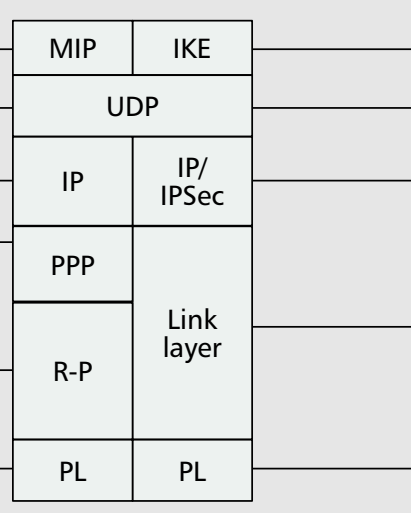

PDSN

\begin{tabular}{|c|c|}
\hline IKE & MIP \\
\hline UDP \\
\hline IP/IPSec \\
Link \\
layer \\
\hline PL \\
\hline
\end{tabular}

HA

IP: Internet Protocol

HA: Home agent

MAC: Medium access control

MS: Mobile station

PPP: Point-to-Point Protocol

RN: Radio network

UDP: User Datagram Protocol

In both control and user

planes for cdma2000,

PPP is carried over the

LAC/MAC and R-P

tunnels and utilized to

establish connection

between the MS and

the PDSN. The PPP

provides a standard

method for transporting

multi-protocol datagrams

(e.g., IP) over

(a)

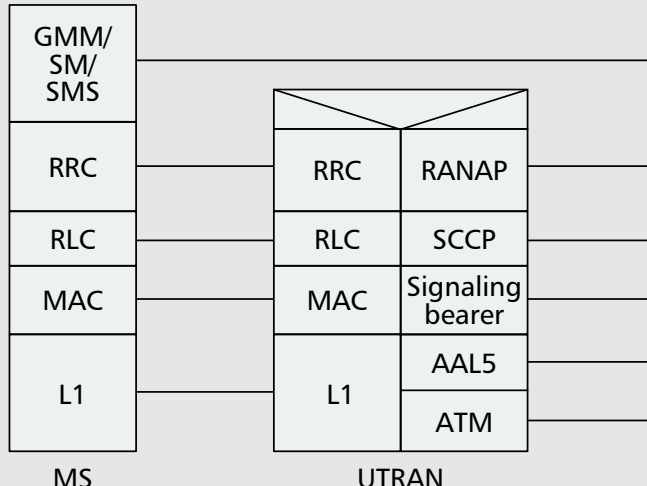

MS

UTRAN

ATM: Asynchronous transfer mode

GGSN: Gateway GPRS support node

MS: Mobile station

RLC: Radio link control

SGSN: Serving GPRS support node

GMM/SM/SMS:GPRS mobility management/session

management/short message service

GTP-C: GPRS tunneling protocol - control plane

UTRAN: UMTS terrestrial radio access network

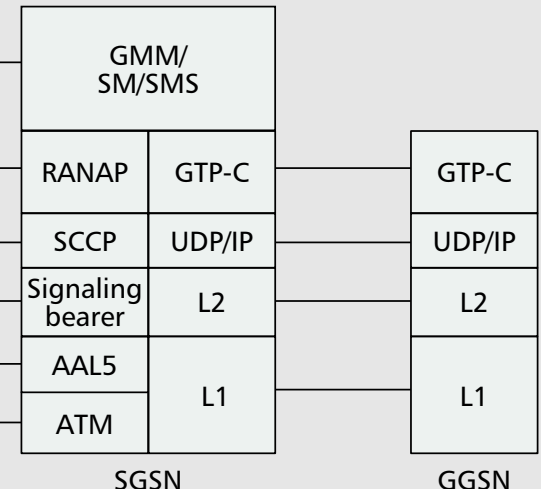

AAL5: ATM adaptation layer type 5

MAC: Medium access control

RANAP: Radio Access Network

Application Part

RRC: Radio resource control

SCCP: Signaling connection control part

(b)

Figure 3. The control planes for a) cdma2000; b) UMTS.

and PDSN. The PPP provides a standard method for transporting multiprotocol datagrams (e.g., IP) over point-to-point links [13]. PPP encapsulates network layer datagrams over a serial communications link, and allows two network nodes to negotiate particular types of network layer protocols (e.g., IP) to be used during a session. In cdma2000, a PPP connection is equivalent to a packet data session, which is comparable to a UMTS PDP context below. After establishing a PPP connection, the MS registers with the MIP HA to indicate its presence and acquire a temporary home address (which is an IP address). The details will be given later.

In the UMTS control plane, no PPP/IP connection is established between the MS and
SGSN. Instead, signaling is carried over the RRC and Iu connections as previously described. The UMTS user plane provides two alternatives for IP services. For the scenario where IP is supported by non-PPP lower layer protocols (specifically, PDCP in the MS and GTP-U in the GGSN), the IP address of an MS is either permanently (statically) allocated or dynamically assigned by the GGSN. Alternatively, the MS's IP address can be assigned by the network nodes outside the GGSN, where the IP protocol is supported over PPP. This alternative is utilized when MIP is introduced to UMTS for global network roaming. In this case the MIP HA is responsible for the MS's IP address assignment. That is, the MS sends the MIP registration mes-

point-to-point links. 
In this case, the MIP HA is responsible for the MS's IP address assignment. That is, the MS sends the MIP registration message to the MIP HA via the PPP connection before obtaining a temporary home address.

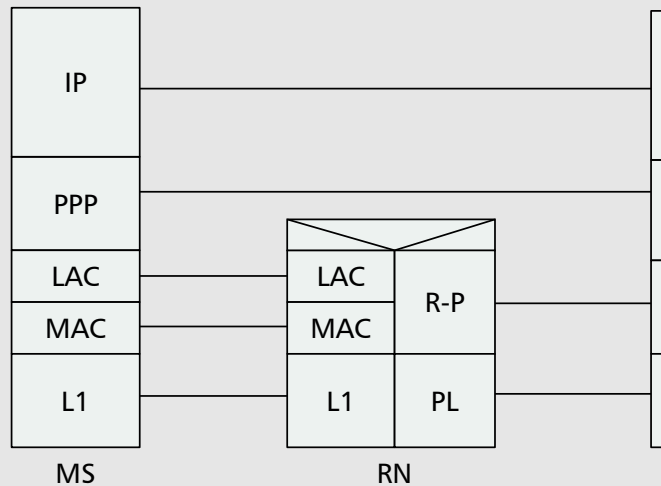

IP: Internet Protocol HA: Home agent MAC: Medium access control PDSN: Packet data serving node PL: Physical layer R-P: RN-PDSN interface

(a)

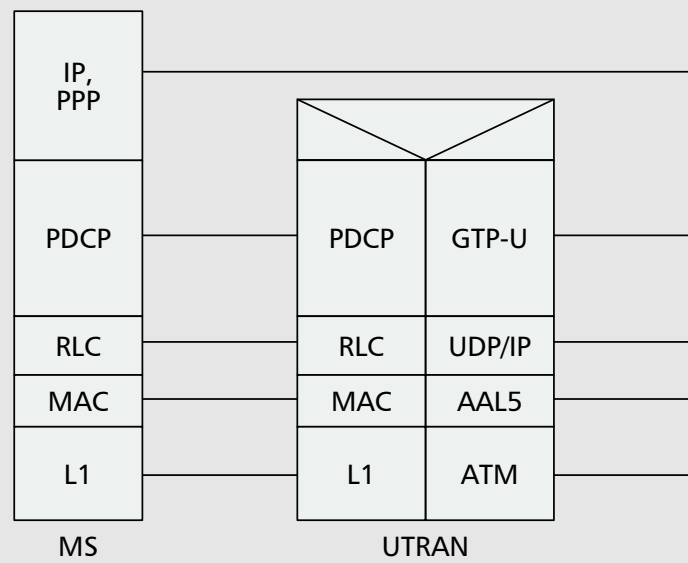

ATM: Asynchronous transfer mode GGSN: Gateway GPRS support node IP: Internet Protocol MS: Mobile station PPP: Point-to-Point Protocol SGSN: Serving GPRS support node UTRAN: UMTS terrestrial radio access network

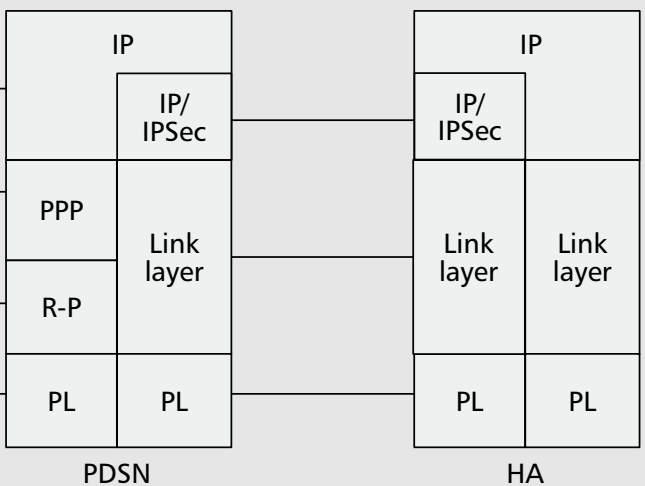

IPSec: IP security

LAC: Link access control

MS: Mobile station

PPP: Point-to-Point Protocol

$\mathrm{RN}$ : Radio network

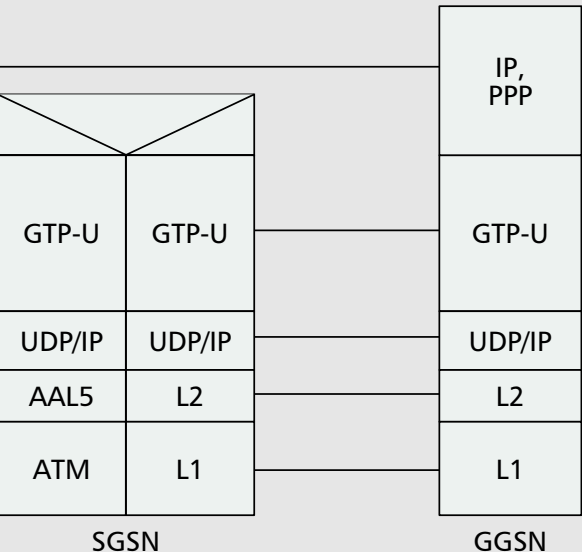

AAL5: ATM adaptation layer type 5 GTP-U: GPRS tunneling protocol - user plane MAC: Medium access control PDCP: Packet Data Convergence Protocol RLC: Radio link control

UDP: User Datagram Protocol

(b)

Figure 4. The user planes for a) cdma2000; b) UMTS.

sage to the MIP HA via the PPP connection before obtaining a temporary home address.

\section{Tunneling Protocols}

In both UMTS and cdma2000, tunneling is used to support various types of mobility. Figure 5 shows the tunneling approaches used in UMTS and cdma2000. In UMTS, the PDCP/RLC/MAC links between the MS and UTRAN support radio link mobility (i.e., handoff). The GTP tunnels between the UTRAN and SGSN, and between the SGSN and GGSN are used to provide serving $\mathrm{RNC}$ relocation [6] and $\mathrm{CN}$ mobility, respectively. On the other hand, cdma2000 utilizes MIP, R-P, and LAC/MAC connections to support IP mobility, $\mathrm{CN}$ mobility, and radio mobility, respectively.

In this subsection we focus on the comparison of CN tunneling implementations for UMTS and cdma2000. GTP is utilized in both the user and control planes of UMTS. In the user plane, the GTP user plane (GTP-U) provides services for carrying user data packets between SGSNs and/or GGSNs, and between an SGSN and an RNC. In the control plane, the GTP control plane (GTP-C) provides GTP-U tunnel-related management and MS mobility-related management between SGSNs and GGSNs.

On the other hand, cdma2000 utilizes a MIPbased tunneling protocol to maintain the R-P connection between PCF and PDSN. We compare UMTS GTP and cdma2000 MIP-based tunneling protocols below.

Tunnel Establishment - In UMTS the SGSN initiates tunnel establishment through the Create PDP Context Request message to the GGSN. In UMTS, every UMTS node (RNC, GGSN, or 


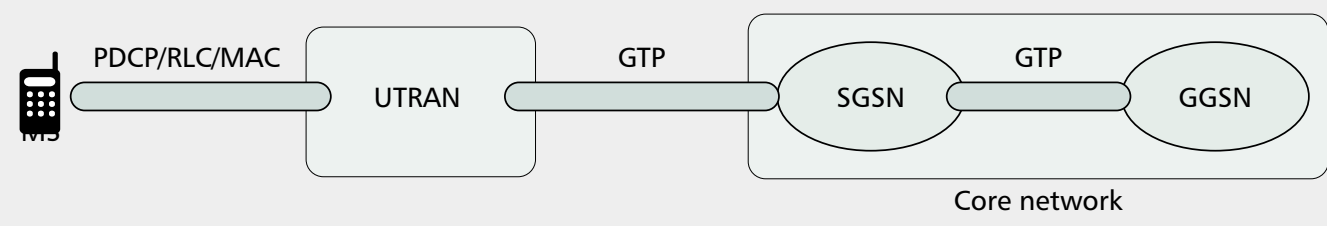

(a)

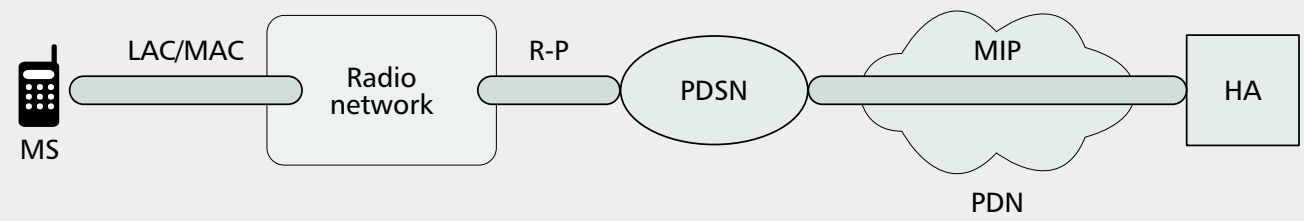

(b)

In UMTS, the base

stations covered by an

SGSN are partitioned

into several routing

areas. When the MS

roams into a new RA,

location update is

performed. Mobility

management in UMTS is

carried out by the

Figure 5. Tunneling approaches for a) UMTS; b) cdma2000.

SGSN) is assigned an IP address. A UMTS GTP tunnel is identified with a tunnel endpoint identifier (TEID), an IP address (i.e., the IP address of the receiving node), and a UDP port number. The TEID is locally assigned by the receiving side of a GTP tunnel, which unambiguously identifies the tunnel endpoint. The TEID values are exchanged between the tunnel endpoints using GTP-C messages.

In cdma2000 PCFs and PDSNs are assigned unique IP addresses. The PCF uses the MIP Registration Request message to initiate R-P connection establishment. In this MIP Registration Request message, the Care-of-Address and Home Agent fields are set to the IP addresses of the PCF and PDSN, respectively. For each packet data bearer, the PCF assigns a PCF session identifier (PSI), which is set in the session-specific extension of the MIP Registration Request message. The PSI, PCF-IP-Address, and PDSN-IP-Address form a unique ID for each R-P connection.

Tunnel Release - The UMTS GTP tunnel release procedure is initiated by either the SGSN or GGSN by issuing the Delete_PDP_Context Request message. Details of this procedure can be found in [6]. Release of an R-P connection in the cdma2000 network is controlled by the PCF. The PCF initiates the R-P connection release procedure by sending an MIP Registration Request message to the PDSN with the lifetime field set to zero (to be elaborated on later). If the PDSN initiates release of an R-P connection, an MIP Registration Update message is sent from the PDSN to the PCF. Then the PCF sends the accounting related information to the PDSN through the normal R-P connection release procedure.

\section{MOBILITY AND Session Management Mechanisms}

As we mentioned in the introduction, the network architectures of mobility and session management are basically the same for both UMTS and cdma2000. However, the protocols exercised on these network architectures are different. Table 1 compares the mobility and session management mechanisms of UMTS and cdma2000. This section will elaborate on mobility and session management mechanisms. These mechanisms are implemented in layer 3 protocols of the control plane; specifically, GPRS mobility management/session management/short message service (GMM/SM/SMS) for UMTS (Fig. 3b), and MIP (over UDP) and PPP for cdma2000 (Fig. 3a). We assume that the reader is familiar with generic mobility and session management operations (e.g., registration, authentication, and session activation, maintenance, and release). For more details the reader is referred to $[1,6,14,15]$. We will focus more on the finite state machines of mobility management and session management. This issue is seldom discussed in the literature.

\section{Mobility Management}

In UMTS, the base stations covered by an SGSN are partitioned into several routing areas (RAs). When the MS roams into a new RA, location update is performed. Mobility management in UMTS is carried out by the mobility management (MM) finite state machine. The MM finite state machines executed in the MS and SGSN are not the same. The details are given in [14]. In this article we only provide common parts of the MS

\begin{tabular}{|l|l|l} 
& UMTS & cdma2000 \\
\hline Protocol (MM) & Mobile Application Part & Mobile IP \\
\hline Involved network nodes (MM) & $\begin{array}{l}\text { Serving GPRS support node and home } \\
\text { location register } \\
\text { Protocol (SM) }\end{array}$ & $\begin{array}{l}\text { Packet data serving node and } \\
\text { home agent }\end{array}$ \\
\hline Involved network nodes (SM) & $\begin{array}{l}\text { Serving GPRS support node and gateway } \\
\text { GPRS support node }\end{array}$ & Point-to-Point Protocol \\
\hline
\end{tabular}

Table 1. A comparison of MM and SM between UMTS and cdma2000. 
Both UMTS and

cdma2000 adopt similar

pipe concepts for session

management. However,

different approaches are

used to implement the

packet data session

between the MS and

the gateway node.
${ }^{3}$ We note that MIP is appropriate for roaming management, but may not support efficient handoff (micro-mobility) management. The micro-mobility issue in cdma2000 is for further study.

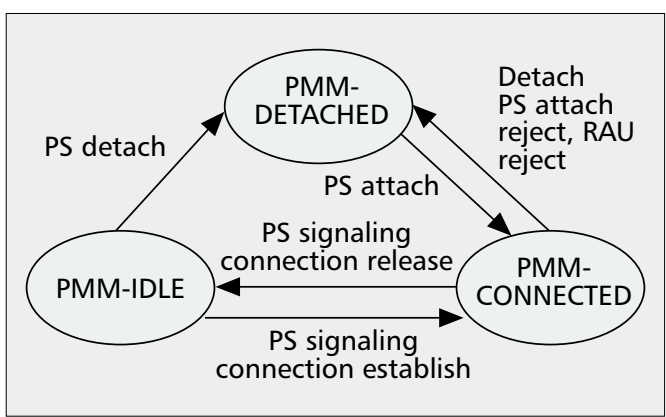

Figure 6. The UMTS mobility management state diagram (common parts in both the MS and $S G S N)$.

and SGSN MM state machines (Fig. 6), which give necessary details to compare UMTS and cdma2000. In Fig. 6 three MM states are defined:

-In the PMM-DETACHED state, the MS does not yet perform a PS attach and is not known to the CN nodes (e.g., SGSN and HLR). When the MS attaches to the PS service domain (e.g., the MS powers on and performs a PS attach), the MM state moves into PMM-CONNECTED.

-In the PMM-IDLE state, the PS signaling connection does not exist; that is, the Iu connection and RRC connection are released. The MS is attached to the PS service domain, and its location is known by the SGSN with accuracy at the RA level; that is, the RA of an MS is tracked by the SGSN. In this state the MS can be reached by paging, and no packet arrives at the MS. The MM state moves into PMM-CONNECTED if packets are exchanged between the SGSN and MS. The MM state moves into PMM-DETACHED if the PS detach procedure is executed.

-In the PMM-CONNECTED state, the PS signaling connection is established. Packets can only be delivered in this state. The SGSN tracks the MS with accuracy at the RA level, and the $\mathrm{RNC}$ is responsible for cell-level tracking. The MM state moves into PMM-IDLE if, for example, no packet is delivered for a period of time. The MM state moves into PMM-DETACHED if the PS detach procedure is executed.

In both the PMM-IDLE and PMM-CONNECTED states, two types of RA update are performed. Normal $R A$ update is performed when the RA of an MS has been changed. Periodic $R A$ update is periodically initiated by an MS to report its "presence" to the network even if the MS does not move. A periodic RA update timer (PRUT) is maintained in the MS. The length of the PRUT is sent from the SGSN to the MS in the Routing Area Update Accept or Attach Accept message. The timer is unique to an RA. Upon expiration of a PRUT, the MS starts a periodic routing area update procedure. Corresponding to the PRUT, a mobile reachable timer (MRT) is maintained in the SGSN. An MRT is slightly longer than a PRUT, and both are stopped in the PMM-CONNECTED state and started in the PMM-IDLE state. Details of mobility management procedures for UMTS Release 99 can be found in [6, 14]. The IP-level mobility for UMTS will be elaborated on below.
In cdma2000 the MIP protocol is utilized for mobility management. ${ }^{3}$ Each PCF corresponds to a packet zone. An FA (PDSN) usually covers one or more packet zones. Location update is performed when the MS roams into a new PCF area. When detecting a change of packet zone in the same FA coverage (i.e., intra-PDSN movement), the MS registers by issuing an Origination Message to the BSC. Then the BSC establishes an A8/A9 connection to the new PCF. When an MS moves into the coverage area of a new FA (i.e., inter-PDSN movement), the MIP registration is activated. Thus, in addition to sending the Origination Message to the BSC, the MS performs MIP registration to the HA by issuing a Registration Request message to its FA. A lifetime value is included in this message. If the registration request is accepted, the HA may grant or change the lifetime value. The negotiated lifetime is included in the Registration Reply message sent to the MS. The MS must perform the re-registration operation before the lifetime expires. Details of cdma2000 mobility management procedures are elaborated on below.

To detach an MS from the cdma2000 network, the MS simply issues a Registration Request with zero lifetime period, which is equivalent to the Detach message in UMTS. As previously mentioned, the MS initiates re-registration before the lifetime expires, which performs the same function as periodic RA update in UMTS.

\section{SeSSION MANAGement}

Both UMTS and cdma2000 adopt similar pipe concepts for session management. However, different approaches are used to implement the packet data session between the MS and the gateway node (i.e., GGSN in UMTS and PDSN in cdma2000). That is, a PPP connection represents a session pipe between the MS and the PDSN in cdma2000. A Packet Data Protocol (PDP) context represents a session pipe between the MS and the GGSN in UMTS, which consists of a PDCP tunnel between the MS and the RNC, a GTP-U tunnel between the RNC and the GGSN, and a GTP-U tunnel between the SGSN and the GGSN.

In UMTS, the PDP context $[6,14]$ provides information (specifically, PDP state for session management) to support packet delivery between an MS and the CN.

That is, to support packet routing in a data communication session, the PDP contexts must be created in the MS, GGSN, and SGSN. This action is called PDP context activation. Some properties of the PDP context (e.g., QoS profile) are not found in the PPP connection of cdma2000. Figure 7 illustrates the PDP state diagram for UMTS session management. Two states are defined in the diagram:

- In the INACTIVE state the data service is characterized for a certain PDP address (e.g., IP address) of an inactive subscriber. In this state, the PDP context contains no routing or mapping information related to that PDP address. Therefore, no data can be transferred. The state moves to ACTIVE when the PDP context is activated.

- In the ACTIVE state, the PDP context for the PDP address is activated in MS, SGSN, and GGSN. Specifically, the PDP context contains 
mapping and routing information for delivering PDP protocol data units (PDUs) between the MS and GGSN for that particular PDP address. The state moves to INACTIVE when the PDP context is deactivated or the MM state moves to PMM-DETACHED.

In cdma2000 a session pipe is established between the MS and the PDSN through a PPP connection. The session management for cdma2000 is similar to that for UMTS. That is, the session states are either open or closed. In the open state, a PPP connection is established between the MS and the PDSN. On the other hand, the logical pipe between the MS and the PDSN does not exist in the closed state. Furthermore, Fig. 8 shows the cdma2000 packet data service state diagram. Three states are defined in this diagram. The relationship between the transmission links (i.e., A8/A10 connections and PPP link) and the packet data service states is elaborated as follows:

-In the NULL/INACTIVE state, the MS powers off, and the packet services are released. That is, the physical traffic channel, A8/A10 connections, and PPP link are released or not established. The state moves to the ACTIVE/ CONNECTED state when the MS powers on and the packet data services are requested.

-In the ACTIVE/CONNECTED state, a physical traffic channel exists between the MS and the BSC. The PPP link between the MS and the PDSN, A8 connection between the BSC and the PCF, and A10 connection between the PCF and the PDSN are maintained.

The BSC for the MS maintains a packet data inactivity timer. This timer is reset when a nonidle data frame is sent or received. If the timer expires, the traffic channel is disconnected and the state moves to the DORMANT state. When the MS powers off, the state moves to the NULL/INACTIVE state.

-In the DORMANT state, the PPP link between the MS and the PDSN, and A10 connection between the PCF and the PDSN are maintained. No physical traffic channel exists between the MS and the BSC, and $\mathrm{A} 8$ connection between the BSC and the PCF is released. When a packet is destined to the MS in the DORMANT state, the PDSN transmits this packet to the PCF through A10 connection. Then the PCF establishes A8 connection to the BSC. The BSC communicates with the MSC for packet session setup admission. After the MSC has approved the session request, the BSC pages the MS and establishes the traffic radio channel with the MS. At this point, the state moves to the ACTIVE/CONNECTED state. The DORMANT to ACTIVE/CONNECTED state transition may also occur when the MS initiates a packet call reactivation procedure (e.g., a packet data call reconnection is requested by the MS). When the MS powers off, the state moves to the NULL/ INACTIVE state.

From the above descriptions, it is clear that A10/A11 connection and the PPP link are maintained during ACTIVE/CONNECTED and DORMANT states. On the other hand, A8/A9 connection and traffic radio channel are only maintained in the ACTIVE/CONNECTED state. In the DORMANT state we do not expect immediate packet transmission, and the connection

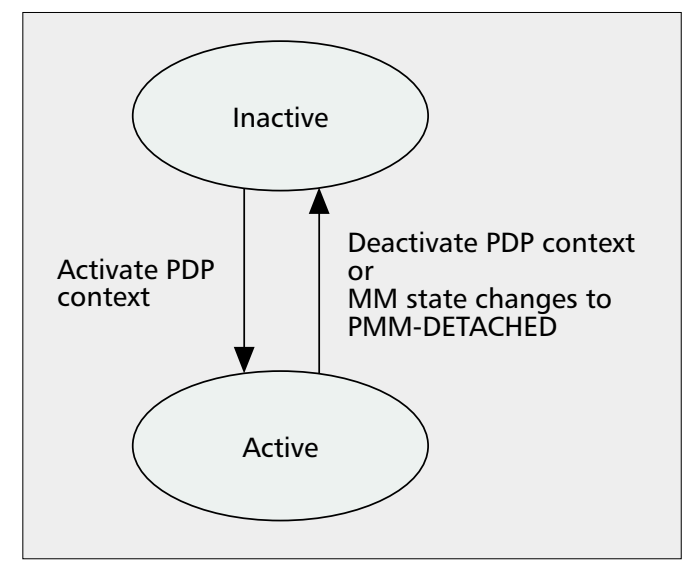

Figure 7. The UMTS PDP state diagram.

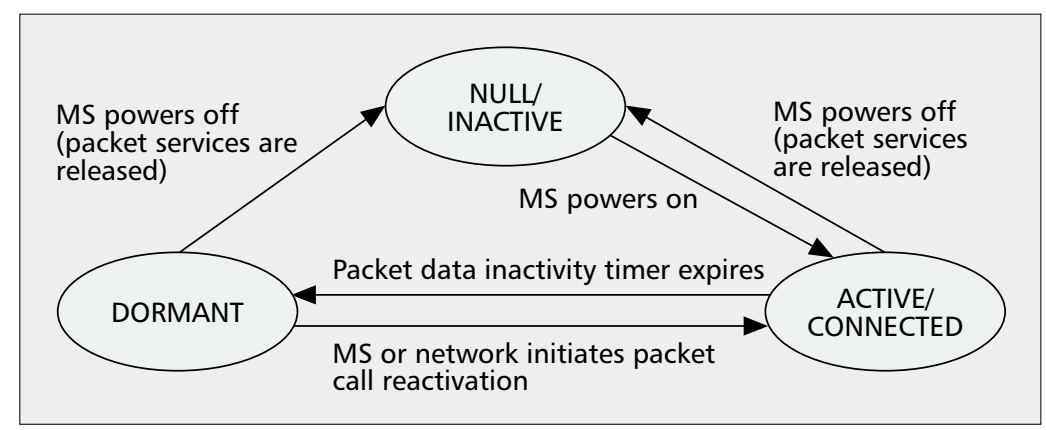

Figure 8. cdma2000 packet data service state transitions.

between the MS and PCF is not established, so the radio resources are not wasted.

\section{REMARKS ON MOBILITY AND SESSION MANAGEMENT}

Mobility management and session management are closely related. For example, if the MS moves across a PCF zone in a cdma2000 network, the old A10/A11 connection is released and the new A10/A11 connection is established. The PPP connection remains the same. If the MS moves across a PDSN area, both PPP and A10/A11 connections are re-established between the MS and the PDSN through the new PCF. If the MS moves to a new PCF zone during a communication session, the A8/A9 connection also needs to be switched from the old PCF to the new PCF.

In UMTS, attachment may or may not be followed by PDP context activation. That is, the session management procedure is independent of the mobility management procedure. This approach provides flexibility for PS domain services. For example, an MS can retrieve the PS domain SMS without activating the PDP context. In cdma2000 MIP registration is always followed by access registration. The advantage of this approach is that the session establishment time can be reduced if a communication session is required immediately after the attach.

As a final remark, we note that UMTS supports multiple PDP contexts (i.e., sessions) simultaneously for a communicating MS. On the other hand, in cdma2000 only one PPP connection can be supported at a time between the MS and the PDSN. 


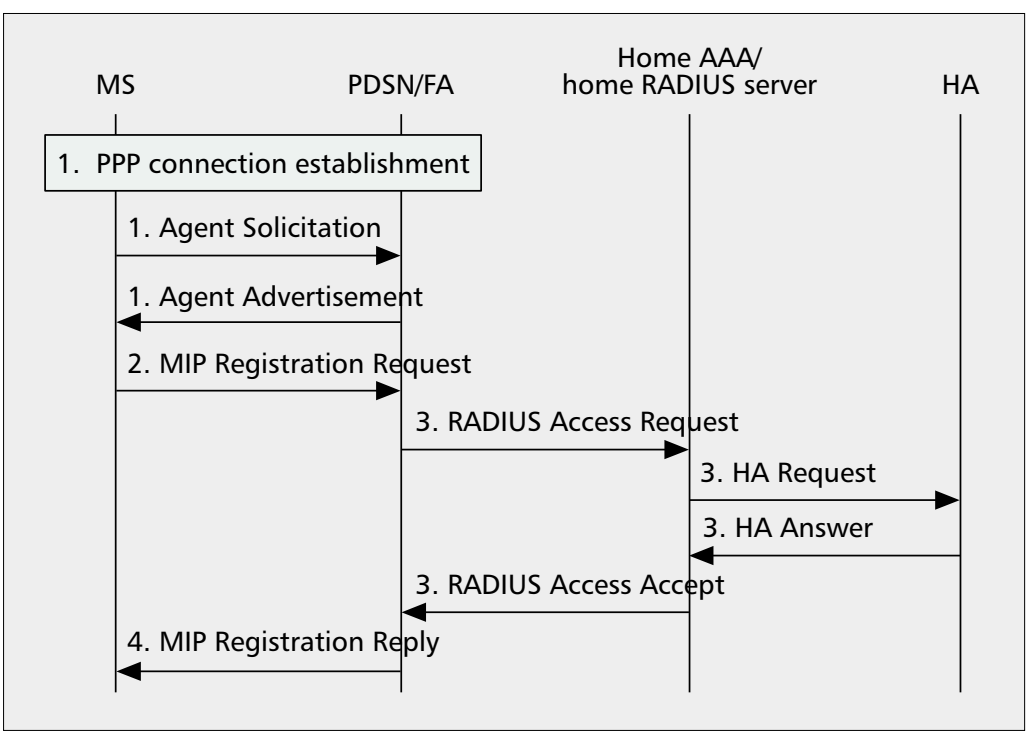

Figure 9. cdma2000 MIP location update procedure.

\section{IP MOBILITY}

As we mentioned in the introduction, IP mobility is different from link layer mobility solutions (e.g., GPRS tunneling protocol vs. MIP-based tunneling protocol). The link layer mobility management function is used to manage tunnels between the CN nodes (e.g., between the SGSN and the GGSN, or between the PCF and the PDSN). On the other hand, the IP mobility mechanism allows the mobile user to change its point of IP connectivity without losing ongoing sessions.

In cdma2000, two types of IP mobility are supported: Simple IP and Mobile IP. In Simple IP, IP address mobility is supported within a PDSN, but is not supported when the MS moves to a new PDSN. The IP address is dynamically assigned by the local PDSN. Challenge Handshake Authentication Protocol (CHAP) [16] or Password Authentication Protocol (PAP) [17] is used for authentication between PDSN and a Remote Authentication Dial In User Servic (RADIUS) server. In Mobile IP, both intraPDSN and inter-PDSN IP address mobility are supported. In this case, the HA serves as the home router of an MS, which is responsible for location information maintenance and IP datagram tunneling of the MS. The HA interacts with AAA to provide a security association to the FA (PDSN). Three kinds of AAAs are defined in cdma2000. The visited $A A A$ forwards the AAA request from the PDSN to the home or broker AAA based on the MS network access identifier (NAI). The broker $A A A$ provides secure AAA message delivery between the visited and home AAAs. The home $A A A$ authenticates the PDSN request based on NAI. The home AAA also supports QoS services based on an AAA profile (that contains a differentiated service policy and HLR record). FA challenge for authentication is performed through the MN-AAA challenge extension procedure [19]. Note that in Mobile IP neither CHAP nor PAP is used because these protocols result in longer initial setup time due to additional RADIUS traversal.
In Mobile IP the IP address of an MS is statically or dynamically assigned by the HA. This persistent IP address is maintained when the MS moves around PDSNs. The location update procedure is illustrated in the following steps (Fig. 9).

Step 1 - When an MS moves into a new PDSN (MIP FA), the PPP connection is established between the MS and the PDSN. Then the agent discovery procedure is exercised. Specifically, the MS sends Agent Solicitations and the PDSN transmits Agent Advertisements. Note that unlike the standard MIP environment, the MS is known by the PDSN/FA in cdma2000 during PPP connection setup. Thus, the Agent Solicitations message can be eliminated for efficiency.

Step 2 - Upon receiving Agent Advertisements, the MS initiates the registration procedure by sending an MIP Registration Request to the PDSN.

Step 3 - The PDSN then stops sending Agent Advertisements and issues a RADIUS Access Request to the home RADIUS server (via broker servers if required) to perform FA challenge authentication. If authentication succeeds, the home RADIUS server sends the HA Request message to the HA, which includes the MIP registration request of the MS. The HA validates the registration request and sends a response back to the home RADIUS server. Then the home RADIUS server acknowledges the PDSN by issuing the RADIUS Access Accept message.

Step 4 - The PDSN sends MIP Registration Reply to the MS, and the location update procedure is completed.

UMTS/GPRS provides IP mobility within a UMTS/GPRS network. However, IP mobility for inter-UMTS/GPRS networks (i.e., inter-GGSN areas) cannot be supported. Without the MIPlike solution, an MS must stay within a fixed GGSN area as long as the PDP context is activated. Based on Third Generation Partnership Project (3GPP) Specification 23.923 [19], we use MIP to provide IP mobility for inter-UMTS/ GPRS networks through a three-stage evolution. Stage I allows mobile users to roam between wireless LAN and UMTS using MIP. Stage II supports IP mobility between two UMTS networks where the GGSN is changed during a session. Stage III provides the same mechanism as stage II except that the SGSN and GGSN are combined into one node, which is similar to the cdma2000 solution in Fig. 9. Details of the three stages are described below.

In stage I, the current GPRS bearer transport is maintained to handle mobility within UMTS networks. MIP allows a user to roam between a wireless LAN and UMTS without losing an ongoing session (e.g., TCP), where the GGSN acts as an MIP FA. The access point name (APN) is used to find the desired GGSN, and the MS stays with this GGSN as long as the PDP context is activated. Since MIP is implemented at the application level, all MIP signaling messages are transported over the UMTS/GPRS user plane. Figure 10 shows the MIP registration procedure in stage I, and the steps are described as follows: 


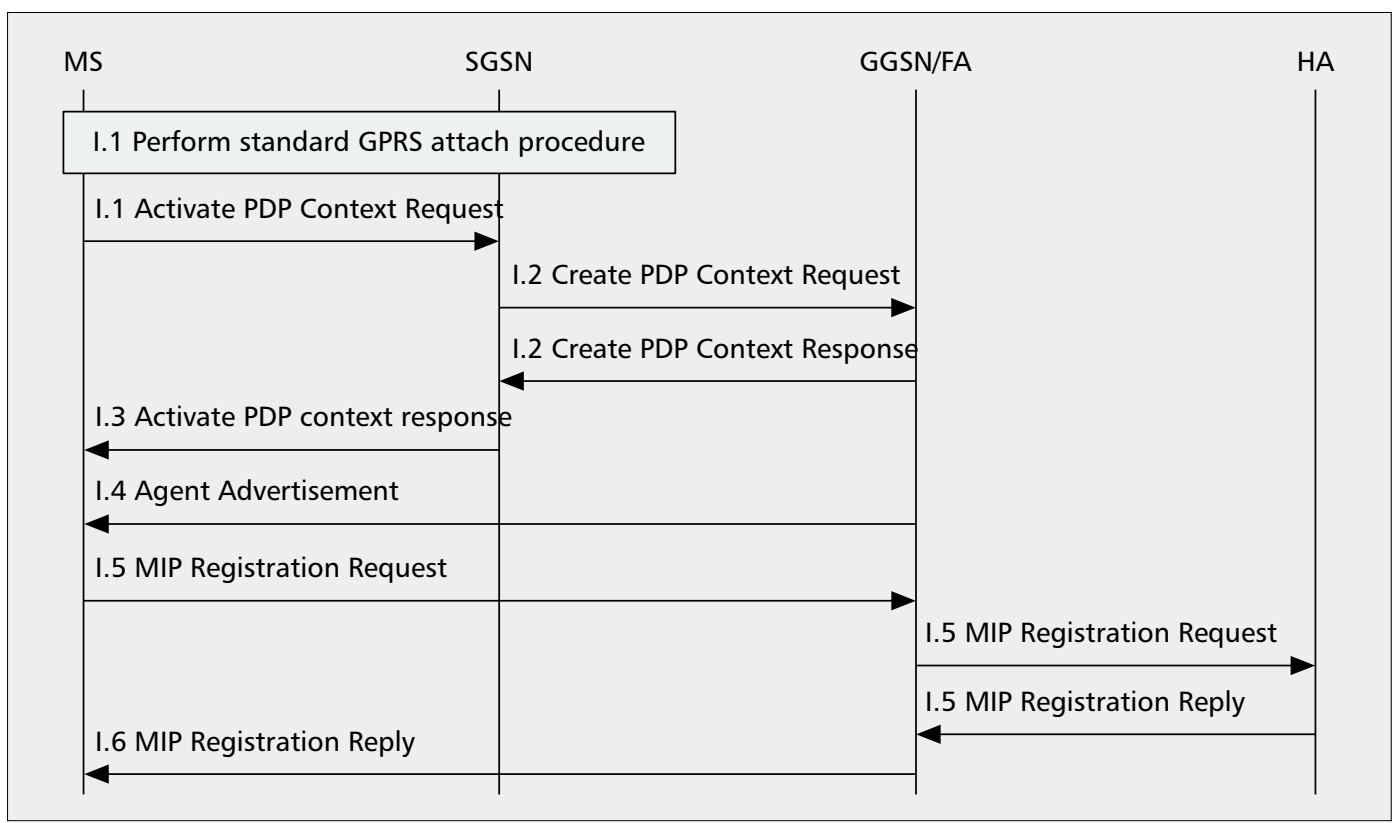

In Stage I, the current

GPRS bearer transport is

maintained to handle

mobility within UMTS

networks. MIP allows a

user to roam between a

wireless LAN and UMTS

without losing an ongoing session, where the GGSN acts as a MIP FA.

Figure 10. UMTS MIP registration: stage I.

Step I.1 - The standard GPRS attach procedure is performed between the MS and the SGSN. Then the MS sends the Activate PDP Context Request message to the SGSN. The APN, MIPv4FA, for MIP registration is included in this message.

Step I.2 - After receiving the Activate PDP Context Request message, the SGSN selects a suitable GGSN based on the APN. The selected GGSN must be equipped with MIP FA capability. Then the SGSN and GGSN exchange the Create PDP Context Request and Create PDP Context Response message pair to set up the MS's PDP context. Note that the GGSN is not responsible for IP address assignment, and the IP address field of the PDP context is not filled at this point.

Step 1.3 - The Activate PDP Context Response message is sent from the SGSN to the MS to indicate that creation of PDP context is successful. At this point, the bearer level connection is established.

Step 1.4 - The GGSN (MIP FA) periodically broadcasts the Agent Advertisement message to announce its presence. When the MS receives this message, step I.5 is executed.

Step 1.5 - The MIP Registration Request message is sent from the MS to the GGSN (MIP FA) across the UMTS/GPRS backbone through the user plane (i.e., the bearer level connection constructed in steps I.1-I.3). The GGSN (MIP FA) forwards the request to the HA. The HA assigns a home (IP) address to the MS and includes this address in the MIP Registration Reply message. Then the message is sent from the HA to the GGSN (MIP FA).

Step $\mathbf{1 . 6}$ - The GGSN (MIP FA) receives the MIP Registration Reply message from the HA and extracts the needed information (e.g., the home IP address of the MS) in this message. Then the MIP Registration Reply message is forwarded to the MS to indicate that the MIP registration procedure is complete.
Steps I.1-I.3 establish GPRS bearer level connection. Then on top of this connection, steps I.4-I.6 perform application level registration (i.e., MIP registration). Also note that the IP address of the MS is assigned by the HA instead of by the GGSN.

In stage II, efficient packet rerouting is supported. The $\mathrm{CN}$ is the same as that in stage I. During a session, the GGSN in the communication path may be changed in two situations:

- After inter-SGSN handoff, the GGSN is changed to optimize the route.

- The GGSN may also be changed for load balancing purposes.

Figure 11 illustrates the scenario for changing a GGSN after changing an SGSN. When changing a GGSN, two tunnels are maintained between the new SGSN and the old GGSN, and between the new SGSN and the new GGSN (Fig. 11c). This will reduce the possibility of packet loss. Figure 12 shows the procedure for changing SGSNs and GGSNs in stage II.

Step II. 1 - After an inter-SGSN handoff, the new SGSN decides to change to an optimal GGSN. The Create PDP Context Request and Create PDP Context Response message pair is exchanged between the new SGSN and the new GGSN to create a new PDP context.

Step II.2 - The HA, GGSN (MIP FA), and MS perform the standard MIP registration procedure as described in steps I.4-I.6 of Fig. 10.

Step II.3 - After successful creation of the new PDP context, a timer is set in the new SGSN. When the timer expires, the new SGSN instructs the old GGSN to delete the old PDP context by sending the Delete PDP Context Request message. The old GGSN deletes the PDP context and responds to the new SGSN with a Delete PDP Context Response message. At this time, the GGSN handoff procedure is complete.

In stage III, the SGSN and GGSN will be combined into one node, called an Internet 


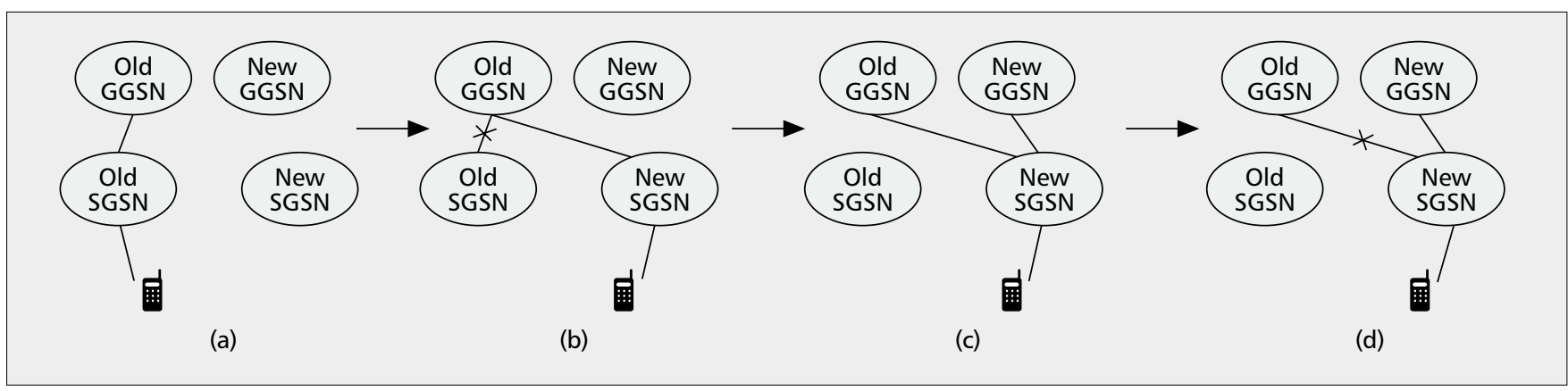

Figure 11. The UMTS scenario for changing SGSNs and GGSNs, stage II: a) before changing the SGSN; b) after changing the SGSN; c) after construction of the second tunnel; d) after changing the GGSN.

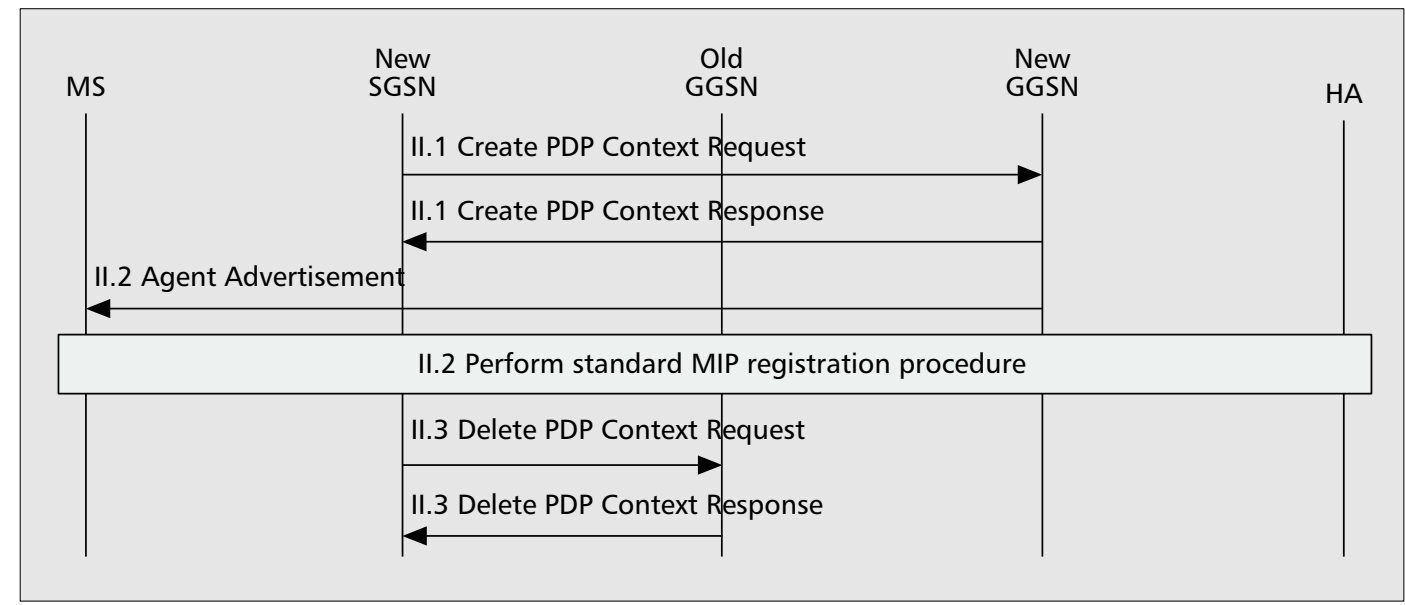

Figure 12. UMTS handoff in stage II.

GPRS support node (IGSN), and MIP is utilized to handle inter-IGSN handoff. The IGSN main functionality includes support of UMTS mobility management across UTRANs, interaction with the HLR, and provision of FA functionality. The details of the IGSN procedures are similar to those in Fig. 10 except that the SGSN and GGSN are merged into an IGSN, and will not be elaborated on in this article. In cdma2000, the Internet AAA functionality is utilized to provide authentication, authorization, and accounting. In UMTS, if the mobile operator and Internet service provider (ISP) are different, AAA is also required when MIP is introduced to provide intersystem IP mobility. ${ }^{4}$ Figure 13 illustrates the network architecture for AAA support of MIP in UMTS. In this figure the MS roams to a visited UMTS network. After the MS has successfully performed UMTS-based authentication with the HLR in the home UMTS network, the AAA mechanism (between the visited and home AAAs) is initiated to authenticate the MS. Then the MS performs MIP registration to the HA in the home ISP to update the location of the MS. For more details of AAA operations in UMTS, the reader is referred to $[19,20]$.

\section{UMTS AND CDMA2000 INTERWORKING}

${ }^{4}$ In the IETF, the $A A A$ working group has incorporated requirements provided by the Mobile IP working group.
UMTS-cdma2000 interworking is essential to support global roaming between these two systems. To achieve complete interoperability between UMTS and cdma2000, the interworking issues at the radio, $\mathrm{CN}$, and IP levels must be addressed. For radio level interworking, a dual mode terminal that contains both UMTS and cdma2000 radio interfaces and the capability to perform real-time handoff among these two systems is required. For $\mathrm{CN}$ interworking, the $\mathrm{MM}$ and PDP contexts have to be migrated between UMTS and cdma2000 networks. These issues are very complex and have not been effectively solved from the business and technical perspectives. We will only focus on the IP level interworking that supports roaming between UMTS and cdma2000 networks. The radio and CN interworking issues are for further study, and will not be elaborated on in this article.

Based on the UMTS and cdma2000 mobility management mechanisms, we propose an architecture and algorithms for UMTS-cdma 2000 interworking to provide real-time IP multimedia services. Figure 14 shows the architecture that interconnects UMTS and cdma2000. In this figure, the UMTS network connects to the IP network through the GGSN that acts as an MIP FA. Following the GPRS attach and PDP context activation procedures described above, the MS performs the MIP registration to its HA via the GGSN (MIP FA). The HA maintains the MS's location information (e.g., the GGSN [MIP FA] address) and tunnels the IP datagrams to the MS. In the cdma2000 network, the PDSN is 


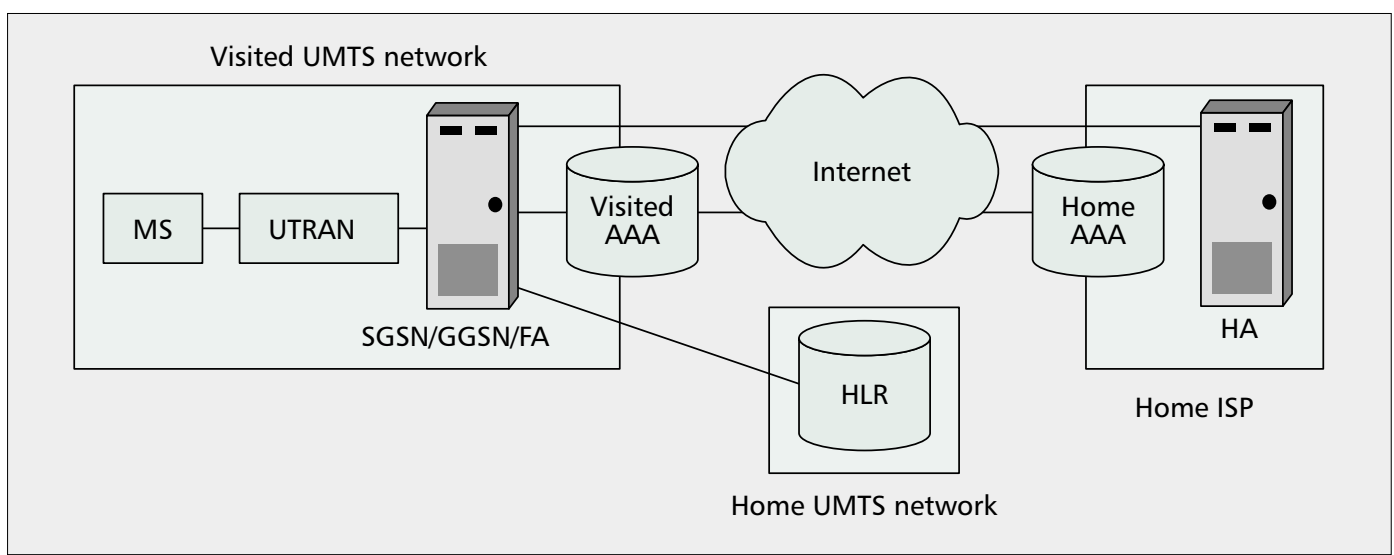

Figure 13. The network architecture for AAA support in UMTS.

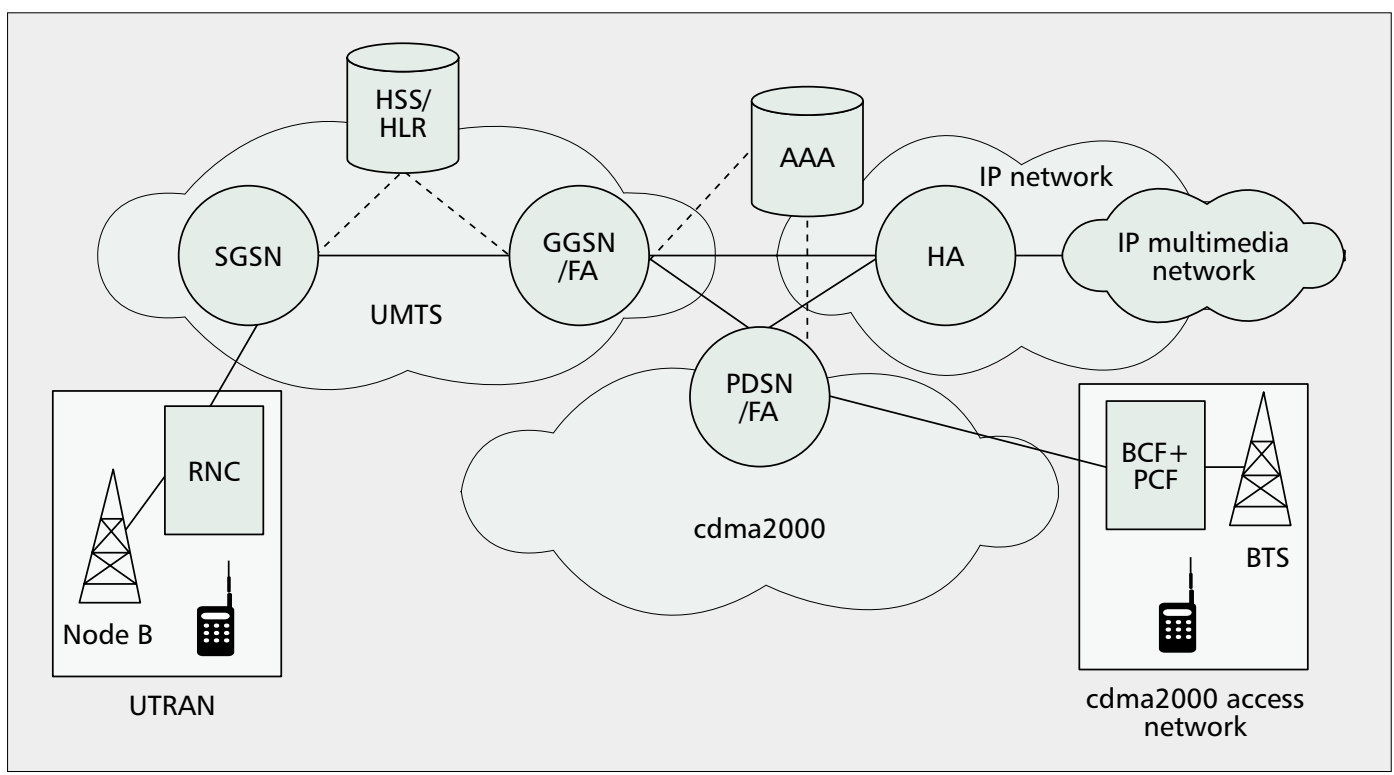

Figure 14. UMTS and cdma2000 interworking.

a MIP FA, which communicates with the HA through the IP network as described previously. Both the UMTS and cdma2000 networks connect to the IP multimedia network through the MIP HA. That is, when a terminal in the IP multimedia network originates a multimedia call to the UMTS or cdma2000 MS by using protocols such as H.323 [21] and Session Initiation Protocol (SIP) [22], the voice packets are first delivered to the MIP HA. Then the MIP HA forwards these packets to the MS through the MIP FA (GGSN or PDSN).

When an MS in communication roams between cdma2000 and UMTS systems, intersystem roaming occurs. We first elaborate on the intersystem roaming message flow from cdma2000 to UMTS (Fig. 15).

Step A.I - The MS performs the standard attach and PDP context activation procedures to register with the UMTS network and set up the bearer communication. This step is the same as steps I.1-I.3 in Fig. 10. Then the AAA procedure is performed at the end of this step.

Step A.2 - After creating the PDP context, the GGSN (MIP FA) issues the MIP Agent Advertisement message to the MS. Upon receipt of the FA advertisement message, the MS performs the MIP registration to the HA via the GGSN (MIP FA). This step is the same as steps I.4 and I.5 in Fig. 10.

Step A.3 - The new FA (GGSN) and the old FA (PDSN) exchange the MIP Binding Update and MIP Binding Acknowledge message pair to perform data forwarding, which provides smooth handoffs between the old and new FAs [11]. Then the PDSN (MIP FA) starts to forward the arriving datagrams to the GGSN (MIP FA). The forwarding operation is used to minimize packet loss during the intersystem roaming procedure.

Step A.4 - The GGSN (MIP FA) responds to the MS's MIP registration request by sending the MIP Registration Reply message. This step is the same as step I.6 in Fig. 10.

Step A.5 - Finally, the PDSN performs standard cdma2000 packet data session clearing operation and de-registration procedure with the $\mathrm{BSC} / \mathrm{PCF}$ and AAA.

The intersystem roaming message flow from UMTS to cdma2000 is illustrated in Fig. 16.

Step B.I - The standard cdma2000 access registration procedure is performed. Then the
Based on the UMTS and

cdma2000 mobility

management

mechanisms,

we propose an

architecture and

algorithms for UMTS-

cdma2000 interworking

to provide the real-time

IP multimedia services. 
After creating the PDP

context, the GGSN

(MIP FA) issues the MIP

Agent Advertisement

message to the MS.

Upon receipt of the FA

advertisement message,

the MS performs the

MIP registration to the

HA via the GGSN

(MIP FA).

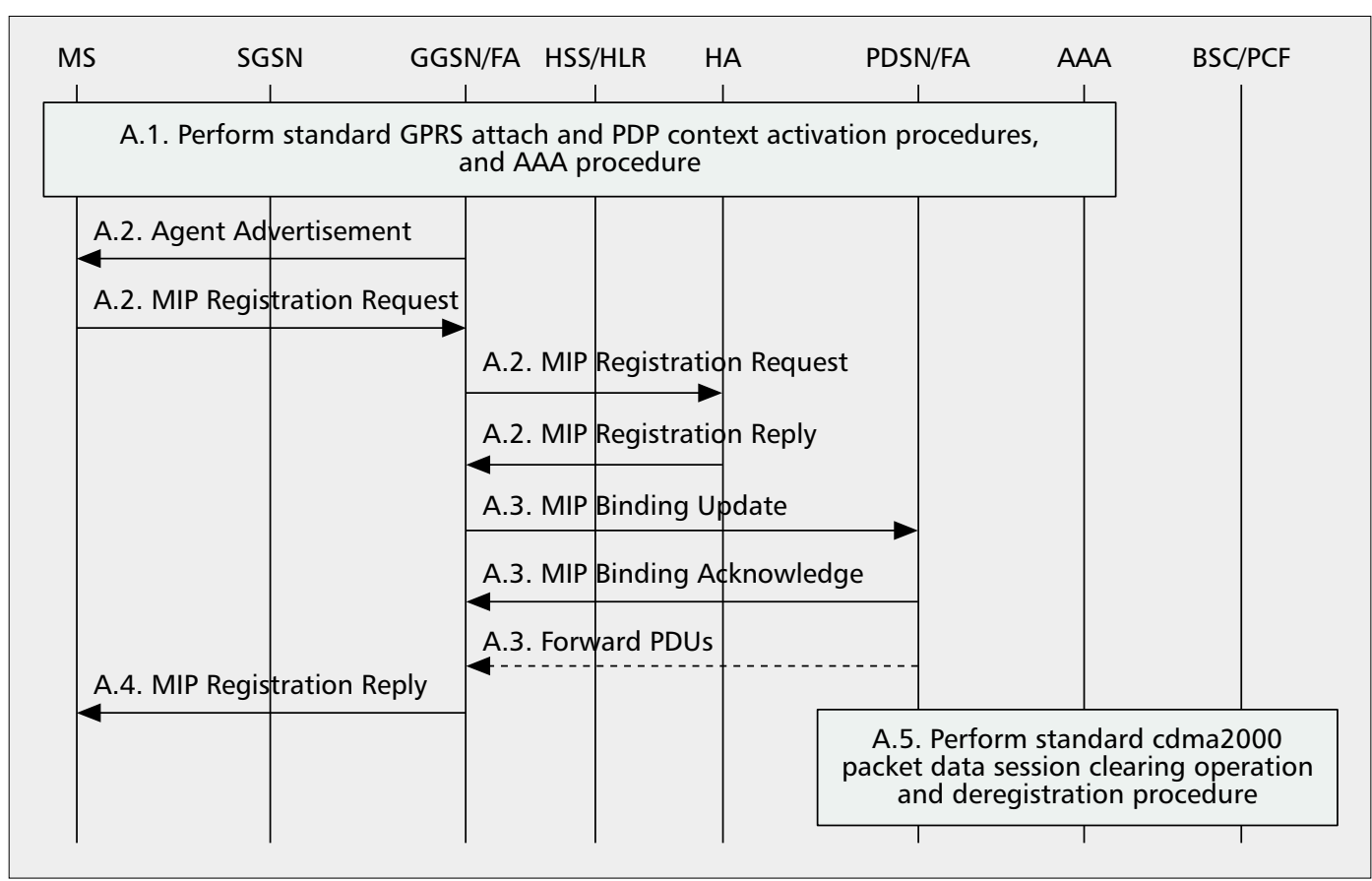

Figure 15. Roaming from cdma2000 to UMTS.

PDSN (MIP FA) and MS exchange the Agent Solicitation and Agent Advertisement to perform the standard MIP agent discovery operation. This is the same as step 1 in Fig. 9.

Step B.2 - Upon receipt of an Agent Advertisement, the MS performs the MIP registration to the PDSN (MIP FA) as described in step 2 of Fig. 9.

Step B.3 - The PDSN (MIP FA), AAA, and HA perform standard authentication and MIP registration procedures. This step is the same as step 3 of Fig. 9.

Step B.4 - The new FA (PDSN) and old FA (GGSN) exchange the MIP Binding Update and MIP Binding Acknowledge message pair as described in step A.3 of Fig. 15.

Step B.5 - Through MIP Registration Reply, the PDSN (MIP FA) informs the MS that the MIP registration is successful. This step is the same as step 4 in Fig. 9.

Step B.6 - When the MRT (discussed earlier) expires, the implicit detach procedure is performed by the SGSN. At this point, the PDP contexts of this MS are also deactivated at the GGSN and SGSN.

\section{SUMMARY}

This article describes mobility and session management mechanisms for UMTS and cdma2000, and compared the design guidelines for these two $3 \mathrm{G}$ technologies. We first introduce the network architectures and protocol stacks for UMTS and cdma2000. Then we elaborate on UMTS and cdma2000 mobility management and session management, and discuss the differences of the design guidelines between these two systems. Furthermore, the IP mobility mechanisms implemented in UMTS and cdma2000 are demonstrated and compared. Based on the UMTS and cdma2000 mobility management mechanisms, we propose an architecture and intersystem roaming procedures for UMTScdma2000 IP-level interworking. In this approach, the mobile user can roam between UMTS and cdma2000 systems without losing an ongoing communication session.

\section{ACKNOWLEDGMENTS}

Ai-Chun Pang's work was sponsored in part by the National Science Council under contracts NSC92-2213-E-002-049, NSC92-2213-E-002-092 and NSC91-2213-E-002-130, Intel, and Microsoft. Jyh-Cheng Chen's work was sponsored in part by MOE Program for Promoting Academic Excellent of Universities under grant number 89-E-FA04-1-4, National Science Council under grant number 92-2213-E-007-019, and Industrial Technology Research Institute under contract 2F-9303-02.

\section{REFERENCES}

[1] Y.-B. Lin and I. Chlamtac, Wireless and Mobile Network Architectures, Wiley, 2001.

[2] M. Zeng, A. Annamalai, and V.-K. Bhargava, "Harmonization of Global Third-Generation Mobile Systems," IEEE Commun. Mag., Dec. 2000, pp. 94-104.

[3] J.-F. Huber, D. Weiler, and H. Brand, "UMTS, the Mobile Multimedia Vision for IMT-2000: A Focus on Standardization," IEEE Commun. Mag., Sept. 2000, pp. 129-36.

[4] D.-N. Knisely et al., "Evolution of Wireless Data Services: IS-95 to cdma2000," IEEE Commun. Mag., Oct. 1998, pp. $140-49$.

[5] S. Kim et al., "Interoperability between UMTS and cdma2000 Networks," IEEE Wireless Commun., Feb. 2003.

[6] 3GPP, Tech. Spec. Group, Svcs. and Sys. Aspects, "General Packet Radio Service (GPRS); Service Description," Tech. Spec. 3G TS 23.060 v. 5.4.0 (2002-12)\}, 2002.

[7] H. Holma and A. Toskala, Eds., WCDMA for UMTS, Wiley, 2000

[8] 3GPP, Tech. Spec. Group, Core Network, "GPRS Tunneling Protocol (GTP) across the Gn and Gp Interface," Tech. Spec. 3G TS 29.060 v. $5.4 .0(2002-12), 2002$

[9] 3GPP2, "Wireless IP Network Standard," 3GPP2 P.S0001A-1 v. $1.0(2001-07), 2001$

[10] 3GPP2, "3GPP2 Access Network Interfaces Interoperability Specification," 3GPP2 A.S0001-A (2000-11), 2000. 


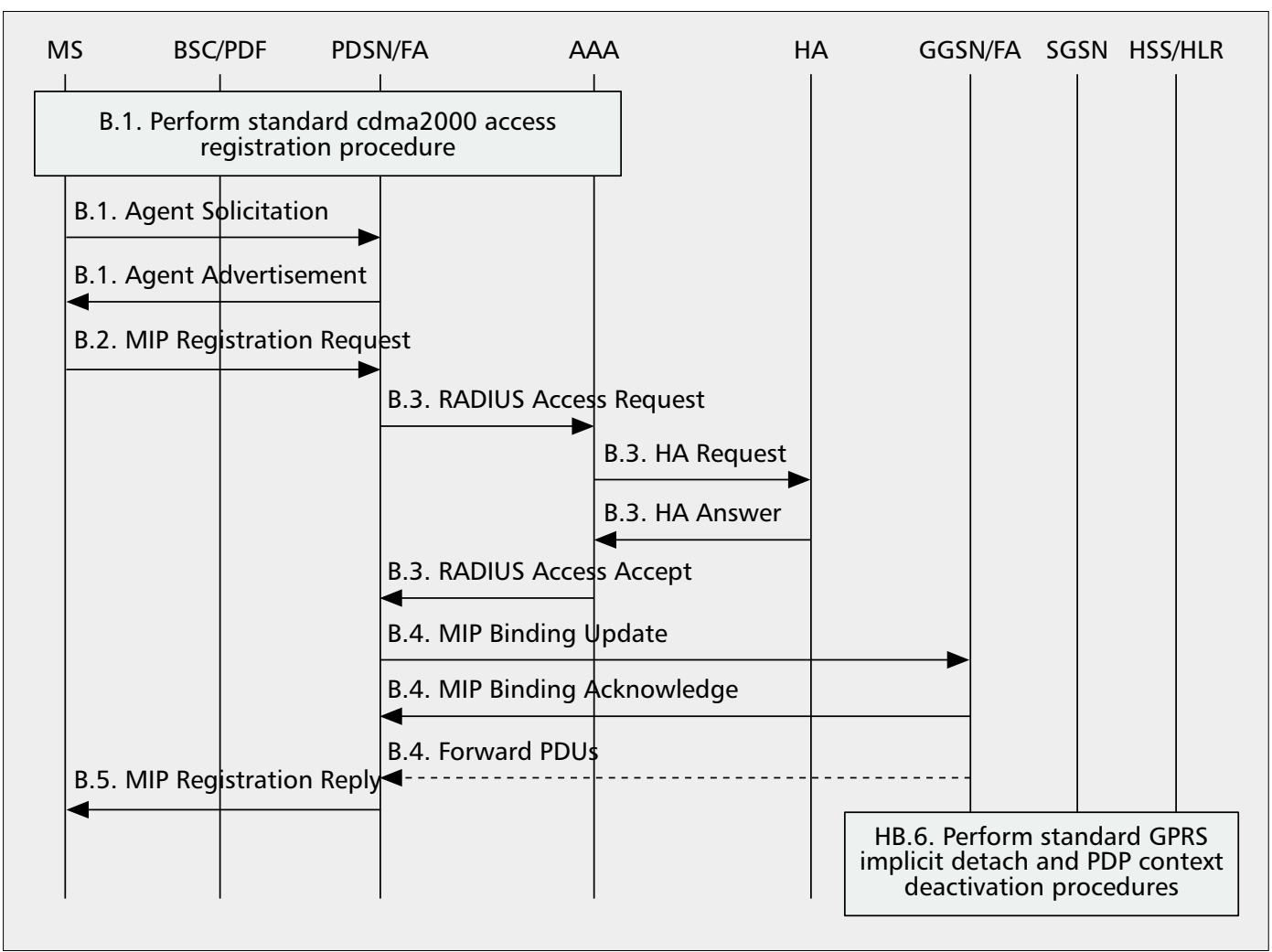

Figure 16. Roaming from UMTS to cdma2000.
Based on the UMTS and

cdma2000 mobility

management

mechanisms, we

proposed an architecture

and inter-system roaming

procedures for UMTS-

cdma2000 IP-level

interworking. In this

approach, the mobile

user can roam between

the UMTS and cdma2000

systems without losing

the ongoing

communication session.
[11] C. E. Perkins, Mobile IP: Design Principles and Practices, Addison-Wesley, 1998

[12] 3GPP2, "IP Network Architecture Model for cdma2000 Spread Spectrum Systems," 3GPP2 SC.P000X v. 1.0.0 (2000-10), 2000

[13] W. Simpson, The Point-to-Point Protocol (PPP), IETF RFC 1661, July 1994.

[14] Y.-B. Lin et al., "Mobility Management: From GPRS to UMTS," Wireless Commun. and Mobile Comp., vol. 1, no. 4, 2001, pp. 339-60.

[15] Y.-B. Lin et al., "All-IP Approach for Third Generation Mobile Networks," IEEE Network, vol. 16, no.5, 2002, pp. 8-19.

[16] W. Simpson, "PPP Challenge Handshake Authentication Protocol (CHAP)," IETF RFC 1994, Aug. 1996.

[17] B. Lloyd and W. Simpson, "PPP Authentication Protocols," IETF RFC 1334, Oct. 1992

[18] C. Perkins and P. Calhoun, "Mobile IPv4 Challenge/ Response Extensions," IETF RFC 3012, Nov. 2000.

[19] 3GPP, Tech. Spec. Group, Svcs. and Sys. Aspects, "Combined GSM and Mobile IP Mobility Handling in UMTS IP CN," Tech. rep. 3G TR 23.923 v. 3.0.0 (200005), 2000.

[20] J.-H. Park, "Wireless Internet Access for Mobile Subscribers Based on the GPRS/UMTS Network," IEEE Commun. Mag., Apr. 2002, pp. 38-49.

[21] ITU, "Packet-Based Multimedia Communications Systems," Tech. rep. ITU-T H.323, v. 3, 1999.

[22] M. Handley et al., "SIP: Session Initiation Protocol," IETF RFC 2543, Aug. 2000.

\section{BIOGRAPHIES}

Al-ChUn PANG (acpang@csie.ntu.edu.tw) received B.S., M.S., and Ph.D. degrees in computer science and information engineering from National Chiao Tung University (NCTU) in 1996, 1998 and 2002, respectively. She joined the Department of Computer Science and Information Engineering, National Taiwan University, Taipei, Taiwan, as an assistant professor in 2002. Her research interests include design and analysis of personal communications services networks, mobile computing, voice over IP, and performance modeling.

JYH-CHENG CHEN (jcchen@cs.nthu.edu.tw) is an associate professor in the Department of Computer Science and the Institute of Communications Engineering, National Tsing
Hua University, Hsinchu, Taiwan. Prior to joining Nationa Tsing Hua University as an assistant professor, he was a research scientist at Telcordia Technologies (formerly Bellcore), Morristown, New Jersey, from August 1998 to August 2001. He received his Ph.D. degree from the State University of New York at Buffalo in 1998. He is coautho of the book IP-Based Next-Generation Wireless Networks (Wiley, 2004)

YUAN-KAI CHEN (ykchen@cht.com.tw) received his M.S., and $\mathrm{Ph}$.D. degrees in computer science and information engineering from NCTU, Hsinchu, Taiwan, R.O.C., in 1989, 1991, and 2003. In 1991 he joined the Telecommunication Laboratories, Chunghwa Telecom Co., Ltd. where he was involved in the implementation of SONET/SDH multiplexers and the development of an ADSL transceiver. In 1998 he worked in the $3 \mathrm{G}$ trial team. Since then he has been involved in the design of the radio access network, mobile packet-switched data and multimedia services, and the study of mobile network evolution. His research interests include design and analysis of PCS networks, 3G networks, wireless Internet, mobile computing, and performance modeling.

Prathima Agrawal [F] (pagrawal@research.telcordia.com) is assistant vice president of the Network Systems Research Laboratory and executive director of the Mobile Networking Research Department at Telcordia Technologies, Morristown, New Jersey, where she has worked since 1998. She was head of the Networked Computing Research Department in AT\&T/Lucent Bell Laboratories in Murray Hill, New Jersey, where she worked from 1978 to 1998 in various capacities. Concurrently, for several years she was an adjunct faculty member of the Electrical and Compute Engineering Department at Rutgers University. Her research interests are computer networks, and mobile and wireless computing and communication systems. She has published over 150 papers and holds 30 patents. She was the recipient of the Distinguished Member of Technical Staff Award of AT\&T Bell Laboratories in 1985, the Telcordia CEO Award in 2000, and the 2001 SAIC Executive Science and Technology Council Publication Award. She is a member of the ACM. She was the recipient of the IEEE Computer Society's Distinguished Service Award in 1990 and an IEEE Third Millennium Medal in 2000. She chaired the IEEE Fellow Selection Committee from 1998 to 2000. She received her Ph.D. degree in electrical engineering from the University of Southern California in 1977. 